Schmerz 2011 $25: 131$

DOI 10.1007/s00482-011-1028-1

Online publiziert: 20. März 2011

(c) Deutsche Gesellschaft zum Studium des Schmerzes. Published by Springer-Verlag all rights reserved 2011
Th. Sitte

Deutsche Palliativstiftung, Fulda

\section{Spezialisierte ambulante Palliativversorgung}

\section{Problem oder Chance für Hausärzte und Spezialisten?}

Eine sozialmedizinische Originalarbeit in „Der Schmerz“ zu den Erwartungen von Hausärzten an die spezialisierte ambulante Palliativversorgung (SAPV): Gehört sie in diese Zeitschrift? Ich meine: unbedingt ja! Palliativversorgung wurde oft von uns Schmerztherapeuten initiiert, es ergeben sich viele Affinitäten und Schnittmengen, sodass Schmerztherapeuten eine wichtige Rolle in der SAPV zukommt. Ein guter Teil der Symptomkontrolle betrifft Schmerzen, Algesiologie ist multiprofessionell. Wir müssen für eine erfolgreiche Schmerztherapie in hohem Maße mit unseren Zuweisern kooperieren und einiges mehr.

Leider war es über Jahrhunderte ärztliche Haltung, sich von Patient und Angehörigen zurückzuziehen, wenn der Arzt glaubte, dass Heilung nicht mehr möglich sei und der Tod nahen könnte. Das heißt, wir Professionelle überließen die Patienten - auch mit all ihren körperlichen Beschwerden - der Nächstenliebe von Klöstern, Hospizen und Sterbehäusern. Das änderte sich erst im 19. Jahrhunderts, als Hufeland damals seine breite Abhandlung über „Die Verhältnisse des Arztes“ 1806 im „Neuen Journal der Practischen Arzneikunde und Wundarzneiwissenschaft " veröffentlichte:

„Selbst im Tode soll der Arzt den Kranken nicht verlassen, noch da kann er sein großer Wohlthäter werden, und, wenn er ihn nicht retten kann, wenigstens sein Sterben erleichtern."
Ein lesenswertes Plädoyer für eine Medizin der Menschlichkeit, jenseits profitorientierter Technisierung.

\section{Der Hausarzt als der Begleiter des Patienten}

Gerade an ihre Hausärzte haben Patienten meist eine besonders enge und gute Bindung. Und es gibt wohl nur wenige Tätigkeiten im heutigen Gesundheitsbetrieb, die ein so tiefes Gefühl an Zufriedenheit mit der beruflichen Arbeit bringen können, wie es für den Hausarzt eine gute Sterbegleitung bei einem langjährigen Patienten ist. Wenn der betreuende Arzt von den Hinterbliebenen gesagt bekommt, es sei gut so gewesen, wie es kam, findet eine vielleicht jahrzehntelange Arzt-Patientenbeziehung ein gutes Ende. Hier ist es besonders wichtig, für neu hinzukommende Experten, feinfühlig darauf zu achten, Bestehendes nicht zu (zer) stören. Es ist bisher nur wenig bekannt, wo unerwartete Fallstricke auftreten können, wenn ein neues System der professionalisierten Sterbebegleitung parallel zu oder anstelle der bestehenden Strukturen aufgebaut wird. Soziologische Studien und eine intensive Versorgungsforschung sind hier gefragt.

Die für mich erfreulichste Aussage in der vorliegenden Arbeit ist die klare Feststellung, dass die befragten Hausärzte an einer sich ergänzenden Kooperation mit den Spezialisten der SAPV interessiert sind. Eine doch überwältigende Mehrheit in dieser Untersuchung vor Einführung der SAPV sind die rund 4/5 der Hausärzte, die sich eine Versorgung ihrer Patienten gemeinsam mit einem "palliative care team" vorstellen können. Sicher gibt es Voreingenommenheiten, teils begründete, teils unsinnige Widerstände auf beiden Seiten, die mit zunehmender Erfahrung in der Patientenversorgung und im Miteinander vorsichtig abgebaut werden müssen.

- Deshalb oder auch trotzdem lautet mein persönliches Fazit: Gute SAPV ohne Hausarzt ist kaum möglich!
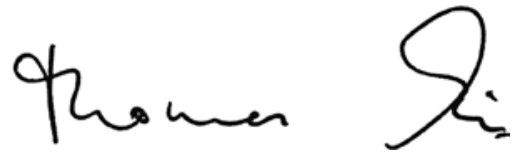

Thomas Sitte

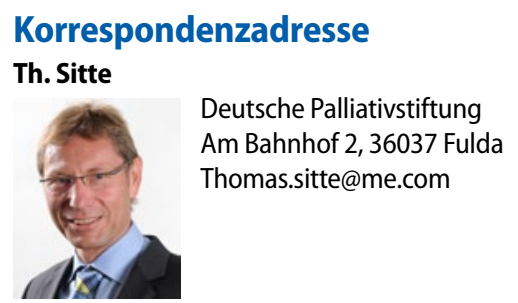

